\title{
Effects of foreground scale in texture discrimination tasks: Performance is size, shape, and content specific
}

\author{
BARTON S. RUBENSTEIN and DOV SAGI \\ Department of Neurobiology, Brain Research, The Weizmann Institute of Science, Rehovot 76100, Israel
}

Received for publication 7 September 1993

\begin{abstract}
Textural gradients can be defined as differences across space in orientation and spatial frequency content, along with absolute luminance and contrast. In this study, stimuli were created with gradients of these types to see how changing the size and shape of the foreground region affects the psychophysical task. The foreground regions were designed as clusters of target texels alternating with interplaced background texels (of the same cluster size). This design gives rise to a texture square-wave, with texture frequency defined by the distance from the beginning of one target cluster to the next. It was found that for stimuli with vertical and horizontal Gabor patches, the relationship between the global and local orientation of the foreground region is a critical variable, indicating some global-local interaction. When the global orientation of the foreground region is orthogonal to local target texel orientation, visibility is optimal for high texture frequencies, while for parallel arrangements, low texture frequencies are most visible. The latter result was also found to a lesser degree for tasks involving contrast gradients as well as spatial-frequency gradients, but with no effect caused by varying the global orientation. The results indicate the existence of a second-stage filter that integrates (across space) responses of similar first-stage spatial filters, and then sums the resultant activities with those of orthogonal first-stage filters, whose spatial proximity are to the sides of the local orientation. The size of these integrating mechanisms may extend to more than $7 \mathrm{deg}$, with connections between smoothed activities of filters with orthogonal orientations spanning approximately $1-2 \mathrm{deg}$.
\end{abstract}

\section{INTRODUCTION}

Texture segmentation involves localization of gradients or borders between image regions having different statistical properties. As such, this task must involve local and global processes to accommodate both localization of the border and statistical analysis respectively. Theories of texture segmentation differ in the relative importance given by the two types of processes. Julesz (1962) introduced the texture paradigm into psychophysics, defining the problem in statistical terms, thus using global concepts to account for human performance. Only later, after discovering the limitations of global accounts (Julesz, 1980), along with the introduction of local geometric features into the texture process (Caelli and Julesz, 1978; Julesz, 1981; Beck, 1982), was the emphasis shifted to local processes. However, these local 'features' or 'textons' were assumed to be followed by a global process, which computes their global statistics (Julesz, 1981; Treisman, 1985). Subsequent research assumed a local 'textural gradient' detection stage, thus avoiding this global process (Beck, 1982; Nothdurft, 1985; Sagi and Julesz, 1987), or transferring it to the final decision stage (Rubenstein and Sagi, 1990). These recent theories of texture segmentation assume a few processing stages which are not strictly local. The different processing stages involve integration over different spatial extents, with the range 
of interaction increasing hierarchically. The work presented here attempts to find the importance of scale in texture segmentation tasks. Towards this goal, we designed a new set of texture stimuli with a periodic foreground. These stimuli consist of texture waves, in which foreground and background texels (texture elements) are interchanged periodically. One important property of such stimuli is that the number of foreground texels is invariant over scale (texture frequency) changes.

The design of such a stimulus is rooted in much research that uses filtering models to extract local energy differences within stimuli (Chubb et al., 1989; Landy and Bergen, 1991), and further can predict human performance levels for segregating various textural regions (Caelli, 1985; Fogel and Sagi, 1989; Malik and Perona, 1990; Rubenstein and Sagi, 1990). All of these models employ linear filters (Gabor, or the like) that are numerously convolved at different locations within a stimulus and are tuned to a specific orientation and spatial frequency, and whose responses increase monotonically with luminance contrast. Typically, the resulting filtered images (also called filter-response maps, with distinct features, at specific orientations and spatial frequencies, represented in different maps) are acted upon by a nonlinear operator. Different nonlinear operations include both half- and full-wave rectification of filter outputs (Malik and Perona, 1990; Graham et al., 1992). Also used are a compressive nonlinearity (Caelli, 1985), a summing of the squared outputs of spatially coincident even and odd Gabor filter outputs (energy) (Fogel and Sagi, 1989), or both (Rubenstein and Sagi, 1990). All of these operators, although different, appear to produce fairly similar results. A second stage of filtering has been proposed that acts to average energy across space (to create a spatial-invariant response, which allows power spectra of two neighboring texture regions to be differentiated) and then to extract the larger gradients. This stage, also denoted as a complex channel (Graham et al., 1992), has been substantiated from recent experiments concerning perceived contrast and suprathreshold patterns (Sagi and Hochstein, 1985; Chubb et al., 1989; Cannon, 1990; Polat and Sagi, 1993). This second-stage implicitly involves lateral interactions within filter-response maps and perhaps between maps with different features represented. Different implementations of this stage include a large classical center-surround filter (e.g., DOG filter) (Rubenstein and Sagi, 1990) that acts upon filter responses within each separate map and whose spatial range can extend up to six times the wavelength of the corresponding first-stage linear filter (Sagi, 1990; Polat and Sagi, 1993). Additional competitive connections between maps have also been implemented (Malik and Perona, 1990) along with accelerated nonlinearities (a fourth power) (Landy and Bergen, 1991) with similarly successful results.

The relative success of these distinctly different operators is due to the little research that has explored connections within and between feature maps. Van Essen et al. (1989) showed physiological evidence for connections within maps by studying the visual cortex of the macaque monkey. They showed that an orientation-selective cell responds with the least strength when the surrounding texels were of the same orientation as the target orientation, while for an isolated target or one with orthogonal lines filling the surround, a much larger response was recorded. Polat and Sagi (1993) have recently represented psychophysical results showing threshold levels of focally presented Gabor patches being both suppressed (close range) and facilitated (long range) by spatially flanked Gabor patches of similar orientations positioned 
in alignment. Such interactions are reduced for similar stimuli with patches positioned out of alignment (i.e. when local texel orientation is orthogonal to the virtual line connecting all three texels). Both results imply non-trivial connections between simple cell or first-stage outputs. The proposed experiments attempt to define more robustly the actual design of this second-stage filter, particularly as it relates to texture discrimination tasks. Textural discrimination tasks typically involve some form of preattentive or parallel processing across the entire visual field and thus may be quite different from those described above.

The experiments are designed to test for feature-specificity (i.e. orientation, spatial frequency, and contrast) as well as the spatial extent of the influence between adjacent and neighboring responses, including connections within and between feature maps. To elucidate the inherent structure of this second stage, we have constructed stimuli with localized Gabor texels, whose inter-texel gradients are limited to one feature and whose foreground texels are positioned in such a way as to study parallel and orthogonal interactions between localized responses (i.e. between clusters of texels aligned in parallel or orthogonal to the local orientation of the texel). As will be shown, foreground visibility is feature-specific with orientation gradient tasks giving rise to an asymmetric performance, depending on the existence of parallel or orthogonal interactions. When the global orientation of the foreground region is orthogonal to the local foreground texel orientation, an optimal human performance exists for small foreground texel clusters (high texture frequencies), while when the global orientation of the foreground region is parallel to the local foreground texel orientation, large foreground texel clusters (low texture frequencies) are more visible. In contrast, contrast and spatial frequency gradient tasks are not at all sensitive to this parallel or orthogonal arrangement, and generally show higher performances for the largest foreground texel clusters. As will be discussed, these results support an anisotropic second-stage filter which acts between (for orientation only) and within maps.

\section{METHODS}

\section{Apparatus}

Stimuli were displayed as grey-level modulation on an Hitachi HM-3619A color monitor, using an Adage 3000 raster display system. The video format was $56 \mathrm{~Hz}$ noninterlaced, with $512 \times 512$ pixels occupying a $15.1 \times 15.6 \mathrm{deg}$ visual area. The mean display luminance was $45 \mathrm{~cd} / \mathrm{m}^{2}$ in an otherwise dark, enclosed environment. Stimulus generation was controlled by a Digital Vax 11/750 and the stimulus display by the Adage local processor. Gamma correction was applied using 10-bit lookup tables.

\section{Stimulus}

The objective of the experiments was to determine how changing size and shape of the foreground region would affect human performance levels in texture discrimination/ detection tasks. The tasks were designed to use stimuli with different types of feature gradients, including orientation, spatial frequency, and contrast. The stimuli of all the experiments were created by placing a foreground region in either of two locations, top or bottom. This introduced positional uncertainty. As Fig. 1a shows, the foreground occupied an imaginary strip, $2 \times 14$ texels in size, situated one row from 
(a)

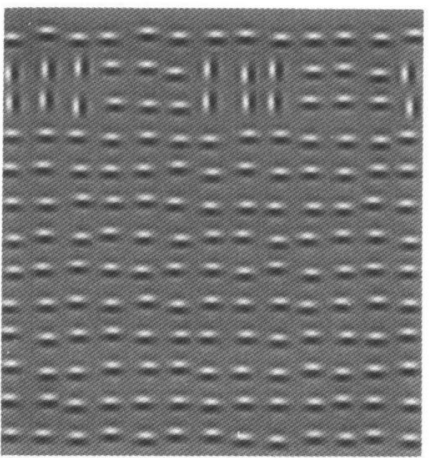

(c)

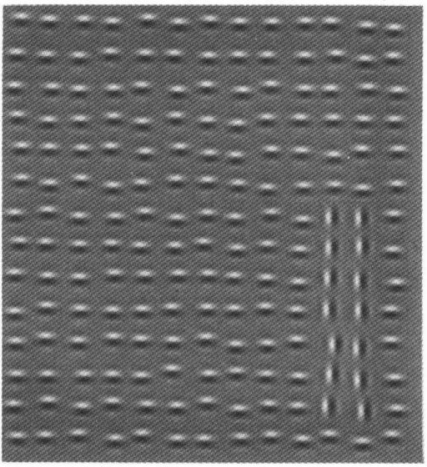

(e)
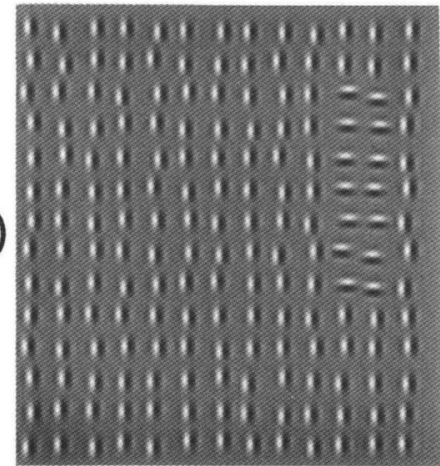

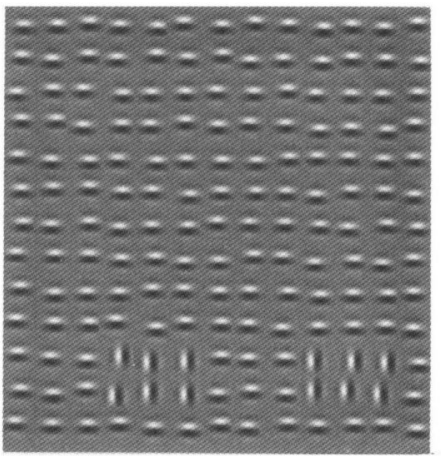

(b)

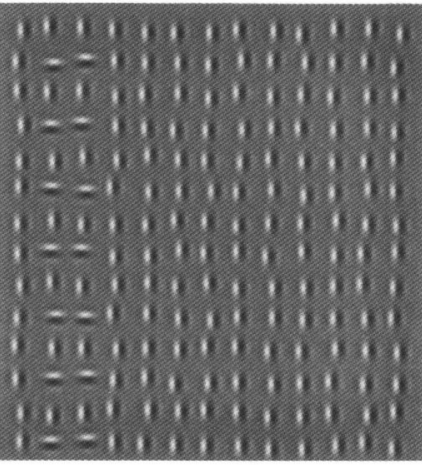

(d)

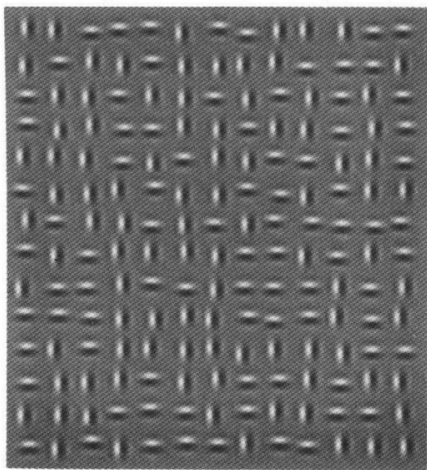

Figure 1. Sample stimuli used in orientation gradient tasks. Texels were presented in a square matrix format, each with positional jitter (up to $0.13 \mathrm{deg}$ in any direction). The foreground was comprised of target Gabor patches (odd-symmetric, $2 \mathrm{cpd}, 50 \%$ contrast, and vertical and horizontal) presented in a squarewave fashion, with clusters of target texels alternated with clusters (same size) of background texels. During a block, stimuli could have the foreground region presented at the top (a) or bottom (b), with the texture frequency (e.g., 1/6 cycles/texel for (a) and 1/14 for (c)) of the foreground region remaining the same, but texture phase changing from trial to trial, i.e. allowing the foreground region to begin (e.g. on the left side) with either target or background texels. The foreground region could also be presented in a left/right format, (c), (d), and (e). Stimulus texels were reversed randomly from block to block. Also, the target texel orientation could be either parallel to the global orientation of the foreground region, (c), or orthogonal (all others), a variable that appears to be critical to the task. A mask was used (f), composed of a $50 \%$ combination of the stimulus texels, but with $100 \%$ contrast. (Note for this figure, higher contrast texels are shown for better visibility.) 
the top (or bottom) edge of a background matrix of $13 \times 14$ texels. The placement of the target texels within this foreground region was such that according to a predefined texture frequency (cycles/texel), target texels were alternated with background texels. As an example, if the texture frequency equaled $1 / 6$ cycles/texel (see Fig. 1a), then the first $2 \times 3$ region of the foreground (starting at the left side) would be occupied with target texels, followed by a $2 \times 3$ region of background texels, followed by a $2 \times 3$ region of target texels, and so on. The phase of this texture square-wave, texture phase, was varied from trial to trial (from 0 to $2 \pi$ ), such that the foreground region could begin with target or background texels (compare Fig. 1a to 1b). Thus, regardless of the texture frequency, a foreground region would contain on average the same number of target texels.

The foreground region was also situated in a left/right format (as opposed to top/ bottom). This allowed the foreground region to be vertically oriented (left/right format) or horizontally oriented (top/bottom format) (compare Fig. 1c-e to $1 \mathrm{a}-\mathrm{b}$ ). This variable is equivalent to rotating the monitor $90 \mathrm{deg}$.

This stimulus design was selected for many reasons. First, placing the foreground region in an area of fixed eccentricity from the fixation point allowed it to be processed in the same peripheral region of the retina. Second, introducing a varying texture phase (from trial to trial) ensured that all target texels were on average at a fixed eccentricity. In other words, every position in the foreground region was filled by a target texel with an equal probability, independent of the texture frequency. This was important because observers have a tendency to shift their gaze after a trial has begun and thus, target texels (with a particular texture frequency) that have a tendency to occupy certain areas more than others might become more visible.

Viewing distance was $1 \mathrm{~m}$, producing a visual angle of approximately $14 \times 15 \mathrm{deg}$ for the stimulus display, and a foreground region centered $4.5 \mathrm{deg}$ from the fixation point. Texels were positioned in an imaginary square matrix, with texels separated by $1.07 \mathrm{deg}$. Texels were composed of Gabor patches of 2 or $16 \mathrm{cpd}$ with a Gaussian envelope having a sigma value of $0.25 \mathrm{deg}$. Gabor patches were constructed using $50 \%$ contrast (unless otherwise stated). Within each stimulus, patches were positionally jittered up to $0.13 \mathrm{deg}$ in any direction. Elements were positionally jittered to reduce phase effects and to ensure that large scale first-stage filters could not be significantly activated by the stimulus because generally no adjacent texels were in phase. Odd Gabor patches (phase equal to 0 ) were used (unless otherwise stated) to ensure the absence of a DC signal, which could add unwanted variables (such as creating a luminance detection task) to the stimulus.

Each of the experiments isolated a particular type of texture gradient, so as to study how different features affect the task. The first experiment used an orientation gradient, with vertical and horizontal Gabor patches $(2 \mathrm{cpd}$ ) placed alternately in both foreground and background regions. The second experiment used a contrast gradient, employing vertical, $2 \mathrm{cpd}$, even Gabor patches of $20 \%$ and $50 \%$ contrast. Finally, the third experiment used a spatial frequency gradient, with vertical Gabor patches of 2 or $16 \mathrm{cpd}$.

\section{Procedure}

We used a standard detection task. At the presentation of a fixation cross in the center of the screen, the observer began the trial with the press of a key. Thereupon, a 
grey-level blank field was shown for a variable period $(270 \pm 90 \mathrm{~ms})$, followed by the stimulus ( $18 \mathrm{~ms}$ ), and then by another blank field (defined as the inter-stimulus interval, ISI; $36,54,72,144$, or $180 \mathrm{~ms}$ ). The ISI was used to control the difficulty of the task. Following the ISI, a mask was presented $(108 \mathrm{~ms})$, and finally another greylevel blank field, at which point the observer determined whether there was a target in the stimulus (key 0 for no target, key 1 for target). Stimuli were presented with targets $50 \%$ of the time, giving rise to $50 \%$ correct response for chance performance and $100 \%$ for perfect performance. Auditory feedback, by means of a keyboard bell, was provided upon observer's error immediately after the response. Fifty trials were presented during each block and at least four blocks were performed for each data point. A constant stimulus method was used with the target texel, texture frequency, foreground presentation format (e.g., top/bottom placement), and ISI remaining constant, while positioning of foreground (e.g., top or bottom), texture phase of foreground, and texel jitter varied from trial to trial. Target/background texels remained unchanged during each block (as opposed to mixing stimuli with different combinations of texel-types) in order to reduce uncertainty and for simpler theoretical analysis (introducing uncertainty as for the stimulus type can enforce a decision strategy that integrates over different gradient detectors and thus introduces some complexity to modeling). The mask comprised a combination of the two stimulus texels, but with $100 \%$ contrast (unless otherwise stated), which were scattered randomly (50\% of each) in the $13 \times 14$ matrix (see Fig. 1 ).

The experiments were designed to test performance levels for three separate variables. The first tested for different texture frequencies, $1 / 2,1 / 4,1 / 8$, and $1 / 14$ cycles/texel (with each texel subtending $1.07 \mathrm{deg}$ of visual angle). Second, data were collected for each of the stimulus texels occupying the foreground region (while the other occupied the background). And third, both top/bottom and left/right presentation formats were used to determine whether global orientation of the foreground region had any effect on the results. All results were collected using only one ISI where possible, in order to determine the relative difficulty of the various tasks. When certain tasks were saturated (performance near $100 \%$ correct), other ISIs were also used to determine the shape of that particular curve. Before each block, a sample stimulus was presented in order for the observers to know in advance what type of stimulus would be shown. Thus, the observer could determine before beginning the block whether the target would be presented at the top/bottom or left/right (only one format during a block) and the type of target (e.g., vertical or horizontal). This was done to reduce stimulus uncertainty. Also, the three variables were randomly chosen from block to block. Sessions were conducted daily with each being no more than approximately one hour, to avoid any effects caused by fatigue. Before the beginning of the experiments, observers were allowed to practice for up to $1 \mathrm{~h}$ (one session), in order to become comfortable with the task.

\section{Observers}

Six observers, five of whom were undergraduate students, took part in the experiments. The students were not told of the purpose of the experiments and were paid to participate. The first author, BR, was also an observer. All had normal or corrected-to-normal vision. During each of the experiments, three to four observers were used. 


\section{RESULTS}

\section{Orientation gradient tasks}

Experiments were performed using stimuli with vertical and horizontal ( $2 \mathrm{cpd}$ ) Gabor patches (see Fig. 1). Figure 2 shows data from three observers (four were used but only three are shown) with percent correct as the ordinate and the texture frequency as the abscissa. Data from each observer are broken into two graphs with the upper graph relating to stimuli using the top/bottom foreground presentation format (horizontally oriented) and the lower graph relating to stimuli using the left/right format (vertically oriented). Depending on which of the Gabor patches is presented in the foreground, the local orientation of the Gabor patch can be parallel or orthogonal to the global orientation of the foreground region (see Fig. 1c and e, respectively). This distinction is made because the data indicate that parallel-vs-orthogonal is a critical parameter which dictates the shape of the curve. For a global foreground orientation parallel to the local Gabor orientation (dotted curves), performance decreases as texture frequency increases. In three cases the thick dotted curves show performance saturation near the $100 \%$ correct level (lower graph of RC, upper graph of BR), thus indicating a task easier than the other task (solid curves). In these cases, ISI was reduced to determine the true shape of the curve, as indicated by the thin dotted curves. These curves also clearly show that as texture frequency increases, the task becomes more difficult. This result implies that textures are not

\section{Orientation Gradient Detection Task}

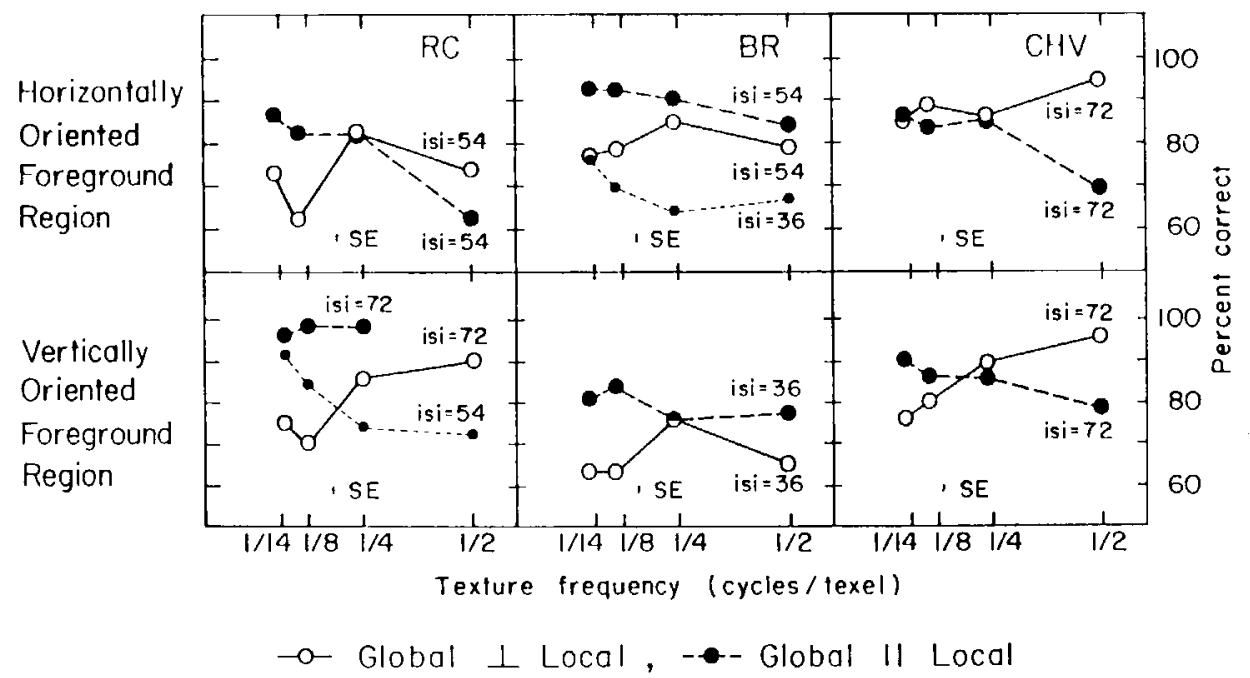

Figure 2. Performance of orientation gradient tasks for three observers. Each observer has two graphs, with the upper graph representing horizontally oriented foreground regions (top/bottom format) and the lower one for vertically oriented foreground regions (left/right format). Correct response is represented as the ordinate and texture frequency as the abscissa. Solid curves represent stimuli with target texel orientation orthogonal to the global orientation of the foreground region. Target texel orientations in parallel are represented by dotted curves. Notice that all solid curves have peak performance around 1/ 2-1/4 cycles/texel (positive slopes), while dotted curves show performance decreasing as texture frequency increases (negative slopes). 
necessarily processed locally; rather, more distant neighbors (i.e. not just adjacent Gabor patches) somehow influence visibility of foreground texels. (If only local connections between adjacent filters existed, then performance would remain constant across texture frequency because the number of target texels remains the same.) In contrast, when the global foreground orientation is orthogonal to the local Gabor orientation, the higher texture frequencies $(1 / 2-1 / 4$ cycles/texel) yields maximum performance, with performance dropping off as texture frequency decreases (solid curves).

Single-tailed $t$-tests were conducted to determine the significance of the data. A simple, but conservative approach was used, testing for negative slopes for tasks with global foreground orientation parallel to the local texel orientation (dotted curves), and for positive slopes for tasks with corresponding orthogonal arrangements (solid curves). Linear regression was performed on each curve (four subjects), followed by averaging each of the observers' four slopes (ignoring saturated curves) across all four observers. These averaged slopes are depicted in Fig. 7, along with those of the other experiments. Statistics for the former task of parallel arrangements predicted non-zero negative slopes with $p<0.01$ confidence for stimuli with either horizontally or vertically oriented foreground regions (dotted curves). The latter task of orthogonal arrangements produced non-zero positive slopes with $p<0.01$ confidence for stimuli with either horizontally or vertically oriented foreground regions (solid curves).

\section{Contrast gradient tasks}

The objective of this experiment was to determine how texture frequency affects a task for texels of different contrast ( $20 \%$ and $50 \%$ contrast). The texels chosen were of the same orientation (vertical) and spatial frequency $(2 \mathrm{cpd})$. Gabor patches were evensymmetric and placed in a checkerboard arrangement with adjacent patches of opposite polarity. This was done to ensure that no large first-stage filters could act upon multiple (neighboring) texels. Like positional jitter, this design limits the size of first-stage filters to approximately that of the stimulus texels. Note that unlike all other experiments, odd-symmetric Gabors were not used because during pilot studies, it was noted that neighboring 2 cpd texels of opposite polarity had inter-texel phase properties that were much more noticeable than those of the $16 \mathrm{cpd}$ texels. In contrast, using even-symmetric patches did not give rise to this asymmetric property. Figure $3 \mathrm{a}$ is an example of a stimulus, with higher contrast texels in the foreground region. Figure $3 \mathrm{~b}$ is an example of the mask, comprised of a combination of the stimulus texels.

Performance curves are depicted for three observers in Fig. 4, with a similar format to that in the previous experiment. Stimuli with higher contrast target texels are represented by solid curves, while those with lower contrast target texels are represented by dotted curves. The results show clearly that performance either decreases as texture frequency increases or remains flat. Flat curves are only found for high-contrast targets, which indicates that the stimulus has a foreground region already saturated with contrast information, and hence, changing the texture frequency will not affect the task. On the other hand, low-contrast targets offer more difficult tasks (stimulus duration is $90 \mathrm{~ms}$ as opposed to the normal $18 \mathrm{~ms}$; and ISI is significantly larger too, 144 and $180 \mathrm{~ms}$ compared with $54 \mathrm{~ms}$ ) with performance 

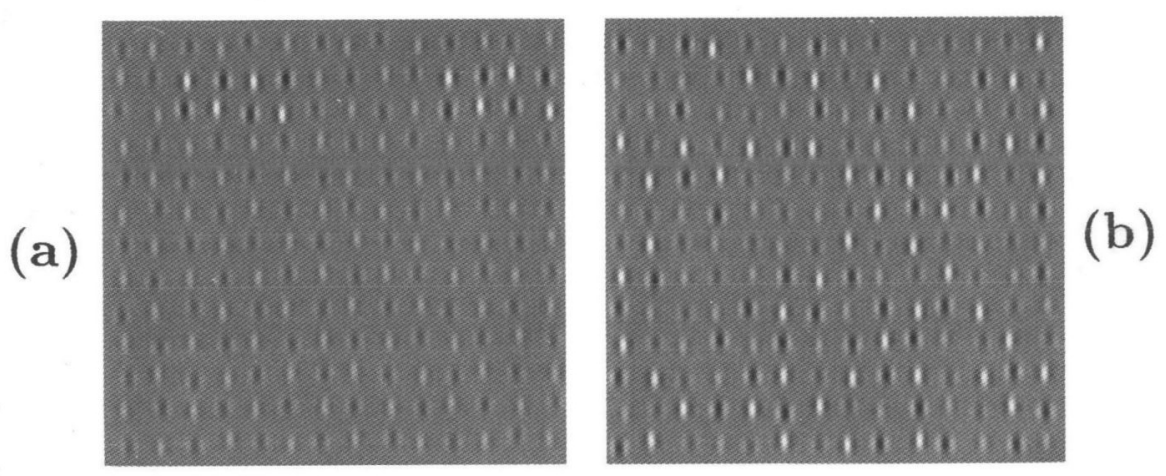

Figure 3. A sample stimulus (a) used in contrast gradient tasks with Gabor patches (even-symmetric, 2 cpd, vertical, and in a checkerboard arrangement with adjacent patches of opposite polarity) of $20 \%$ and $50 \%$ contrast. The mask (b) was comprised of a combination of the stimulus texels. Refer to Fig. 1 for more details. (Note for this figure, higher contrast texels are shown for better visibility.)

levels decreasing as texture frequency increases (also for some observers using highcontrast target stimuli). These results imply some type of integration effect between adjacent and more distant filters (receptive fields) within any particular (orientation or spatial frequency) map.

Single-tailed $t$-tests were conducted to determine the significance of the data. Linear regression was performed on each curve (from Fig. 4), followed by averaging each of the observers' four slopes across all three observers. These averaged slopes are depicted in Fig. 7. Statistics for tasks involving stimuli with low-contrast target texels (dotted curves) show negative slopes with $p<0.01$ confidence while saturated tasks with high-contrast target texels (solid curves) produce slightly negative slopes (no confidence). Collectively, curves have negative slopes with $p<0.005$ confidence.

\section{Spatial frequency gradient tasks}

This experiment was similar to the previous ones, except with spatial-frequency gradients as the only cue used for detection. The experiment used two Gabor patches of 2 or $16 \mathrm{cpd}$ (see Fig. 5a). Both texels were vertically oriented and had identical Gaussian envelopes $(\sigma=0.25 \mathrm{deg})$. The texels were chosen to be three octaves apart so that according to multiple channel theory for spatial frequency content (Blakemore and Campbell, 1969; Graham and Nachmias, 1971), the evoked spatial frequency channels would be significantly separated and thus for the most part act independently. Figure $5 \mathrm{~b}$ is an example of the mask used during this experiment, comprised of a combination of the stimulus texels.

Also, care was taken in choosing the Gabor patches so that they were perceived as iso-contrast. To determine iso-subjective-contrast measurements for both texels, we ran a two-alternative forced-choice (2AFC) pre-experiment before each day's session. During this pre-experiment, the observer was asked to determine which side (left or right) was brighter, with each side composed of only one type of texel ( 2 or $16 \mathrm{cpd})$. Observers were asked to press key 1 for a left-brighter stimulus and key 0 for a right-brighter one. The placement of a texel-type on a particular side was randomized $(50 \%)$ from trial to trial. Using a staircase method, one texel $(16 \mathrm{cpd})$ had a constant contrast of $80 \%$ while the other varied during the course of the block. Like all 


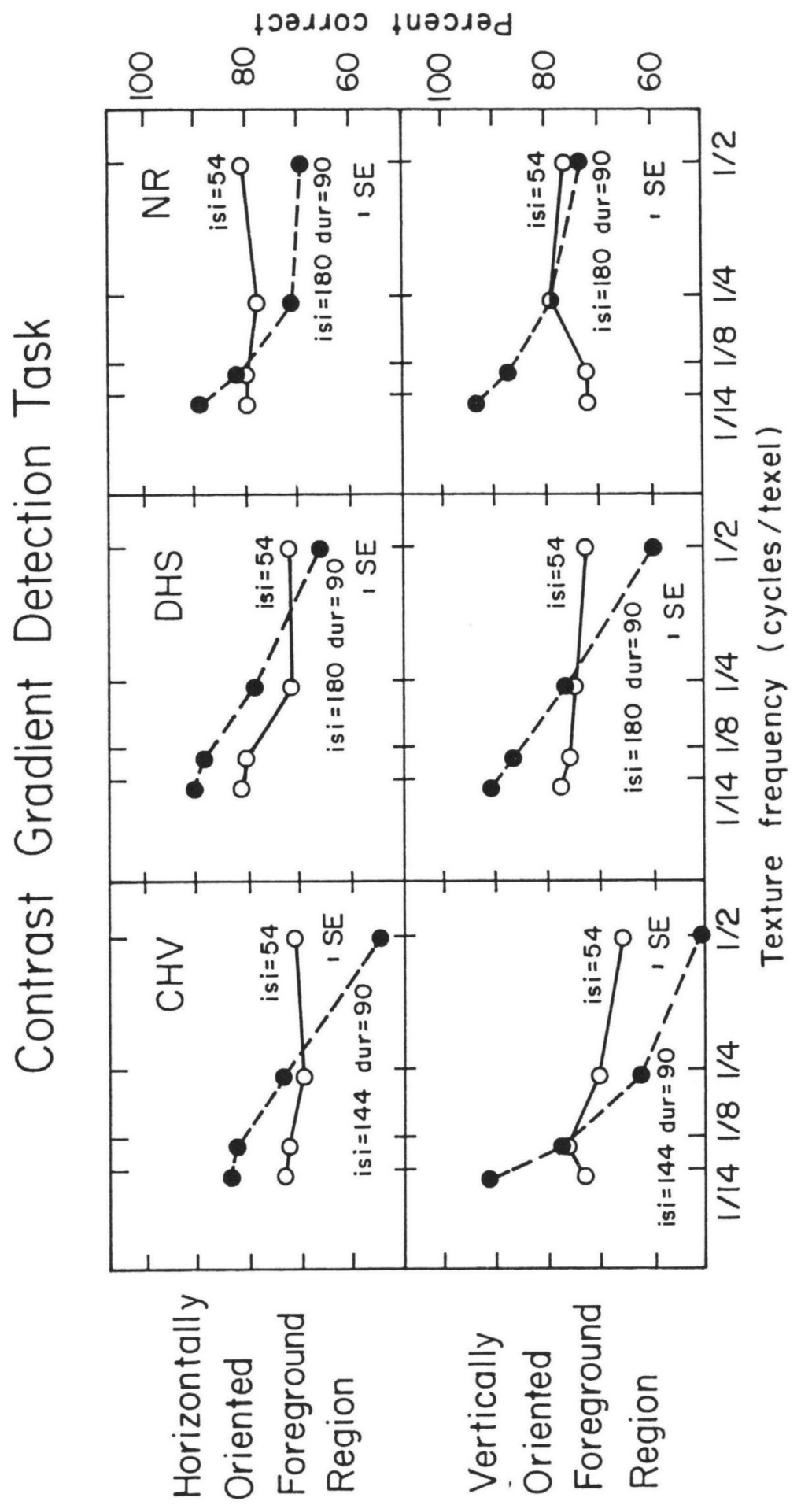

蛋 용 ถิ 宥.马 尤它 온 苞 象 (1) O) क्षे 응ํำ 七 을

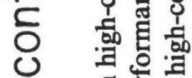

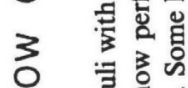
है क्

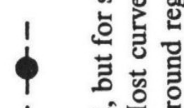
त) 语遅 . ๑

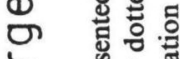
○े 힘 - 용잉 음

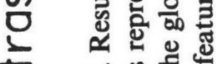

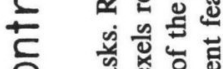
잉

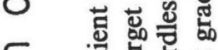

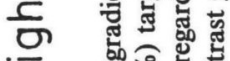

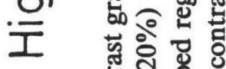
1 焉空考

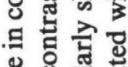
ช ํㅠㄹㅠ 능은

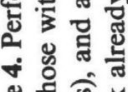
至路

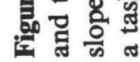



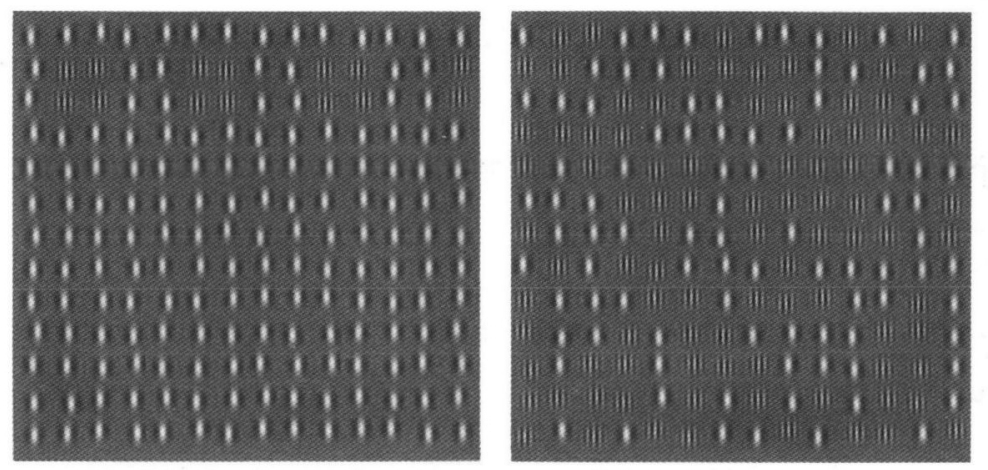

Figure 5. A sample stimulus (a) used in spatial-frequency gradient tasks with Gabor patches (even-symmetric and vertical) of $2 \mathrm{cpd}(20 \%$ contrast) and $16 \mathrm{cpd}$ ( $80 \%$ contrast). Contrast values were chosen so that texels were iso-subjective contrast. The mask (b) comprised a combination of the stimulus texels. Refer to Fig. 1 for more details. (Note for this figure, higher contrast texels are shown as well as lowering the spatial frequency of the $16 \mathrm{cpd}$ patches for better visibility.)

experiments mentioned, the stimulus was presented for $18 \mathrm{~ms}$, except no mask was used (to simplify the task). One to two blocks, of 60 trials each, were performed each day before the actual experiment. According to Levitt (1971), using this number of trials is sufficient to obtain results that are statistically stable. One of Levitt's methods was used, grabbing mini blocks of four trials and increasing the contrast of the 2 cpd texels (by an absolute $10 \%$ ) if percent correct was above $50 \%$ and reducing the contrast if it was less than $50 \%$ correct. Results from this pre-experiment were both constant across observers and from day to day, with iso-subjective-contrast texels found when the $2 \mathrm{cpd}$ texels were $20-22 \%$ contrast. In light of these results, texels for this experiment were constructed with $20 \%$ contrast ( 2 cpd patches) and $80 \%$ contrast ( $16 \mathrm{cpd}$ patches).

Results are depicted in Fig. 6 for three observers in the same fashion as in the previous experiments. The solid curves represent stimuli with $16 \mathrm{cpd}$ Gabor patches in the foreground region, and the dotted curves represent $2 \mathrm{cpd}$ target texels. All curves show that performance either decreases or remains flat as texture frequency increases. The negatively sloped curves imply some type of integration mechanism that enhances visibility of the foreground region as the texture frequency decreases. In addition, for most curves (except the lower curve of DHS), stimuli with $16 \mathrm{cpd}$ target texels are much easier to see. Notice that observer NR needs an ISI as high as $180 \mathrm{~ms}$ to detect the $2 \mathrm{cpd}$ target texels. This result implies that visibility of spatial frequency information is dependent on which frequency (high or low) occupies the background. Single-tailed $t$-tests were again conducted to determine the significance of the data. Linear regression was performed on each curve (from Fig. 6), followed by averaging each of the observers' four slopes across all three observers. Statistics show a negative slope with $p<0.01$ confidence. These averaged slopes are depicted in Fig. 7, which summarizes all of the results of the experiments.

\section{DISCUSSION}

Psychophysical experiments of texture discrimination tasks were performed to determine the importance of foreground cluster size (defined here as texture frequency, see 


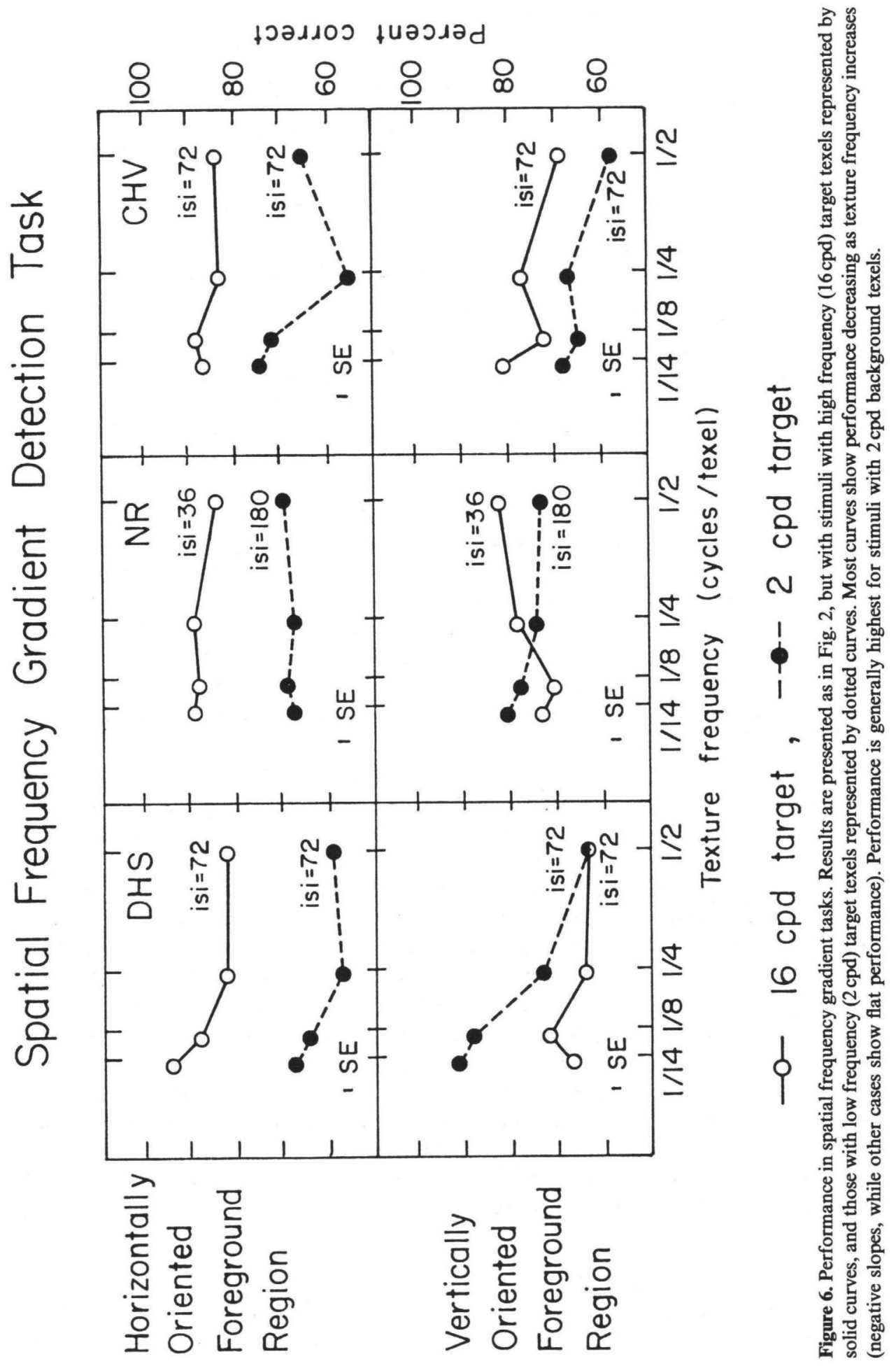




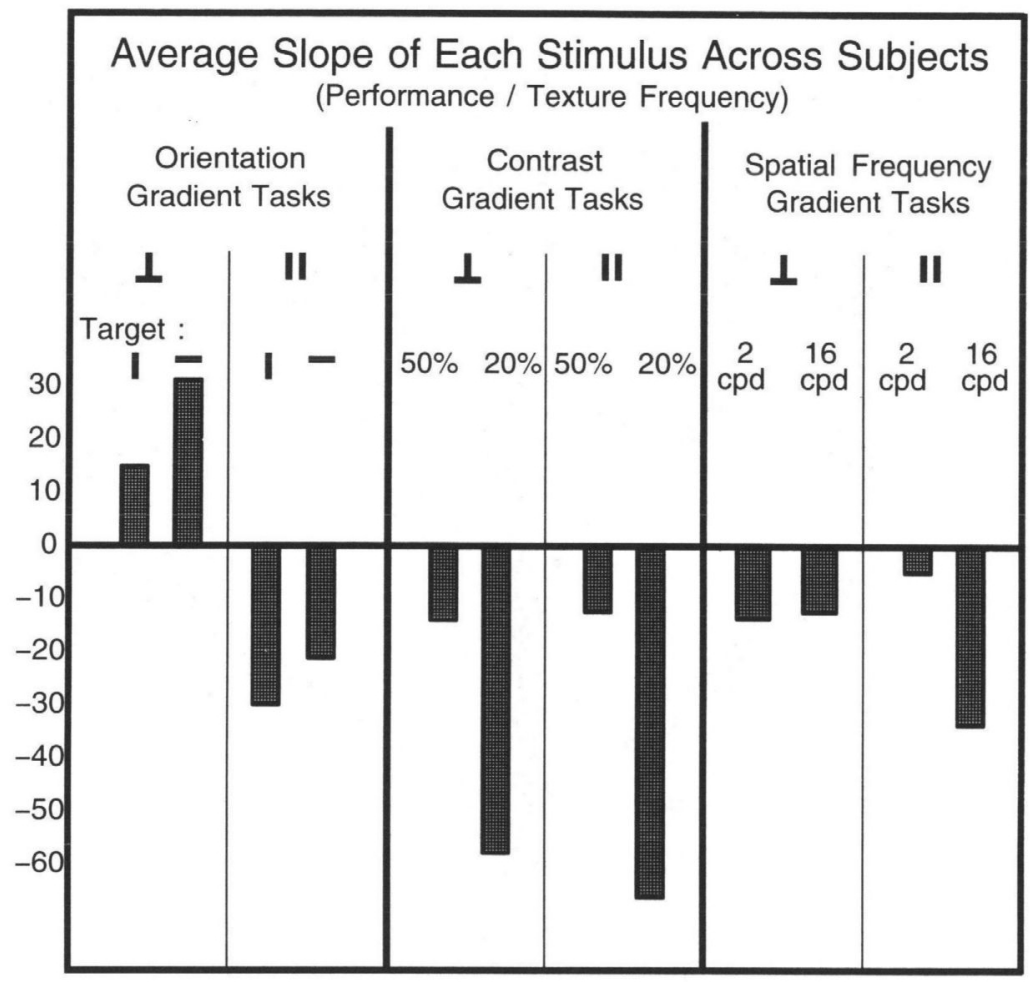

Figure 7. Summary of all results depicted as histograms for each of the three experiments. Within each experiment, a slope of each of the performance curves (Figs 2, 4, and 6) is calculated using linear regression and then averaged across subjects. Each histogram thus represents a slope of each of the corresponding tasks, expressed as performance in percent correct divided by texture frequency. Above each histogram, the target texel is designated. Also, the tasks are grouped so that the foreground texel orientation is either orthogonal or parallel to the global orientation of the foreground region. Notice that slopes are positive only for orthogonal arrangements of orientation gradient tasks.

Methods for formal definition) and shape of the foreground region (global orientation), as related to its surround. The results of this study, as summarized in Fig. 7, show there exist global-local interactions, and that some unique spatial interactions exist within the orientation domain but not within the spatial-frequency domain. For orientation gradient tasks, detection rate was found to be dependent on the relative positioning of the target texels within the foreground region, particularly, if the orientation of the target texels were orthogonal to the global orientation of the foreground region (e.g., Fig. 1a-b, d-e; also see positive slope histograms of Fig. 7). On average, observers performed best when the texture frequency was $1 / 2-1 / 4$ cycles/ texel (e.g., Fig. 1d). As the texture frequency decreased (larger foreground clusters), performance levels dropped rapidly. The opposite behavior was found for target texels aligned in parallel to the global orientation of the foreground region (e.g., Fig. 1c; see negative slope histograms of Fig. 7), with performance levels decreasing as texture frequency increased. Both results imply a neural architecture that is spatially anisotropic, and is dependent on the relative position of neighboring filters (i.e. parallel vs orthogonal arrangements). However, with these results alone, it is uncertain 


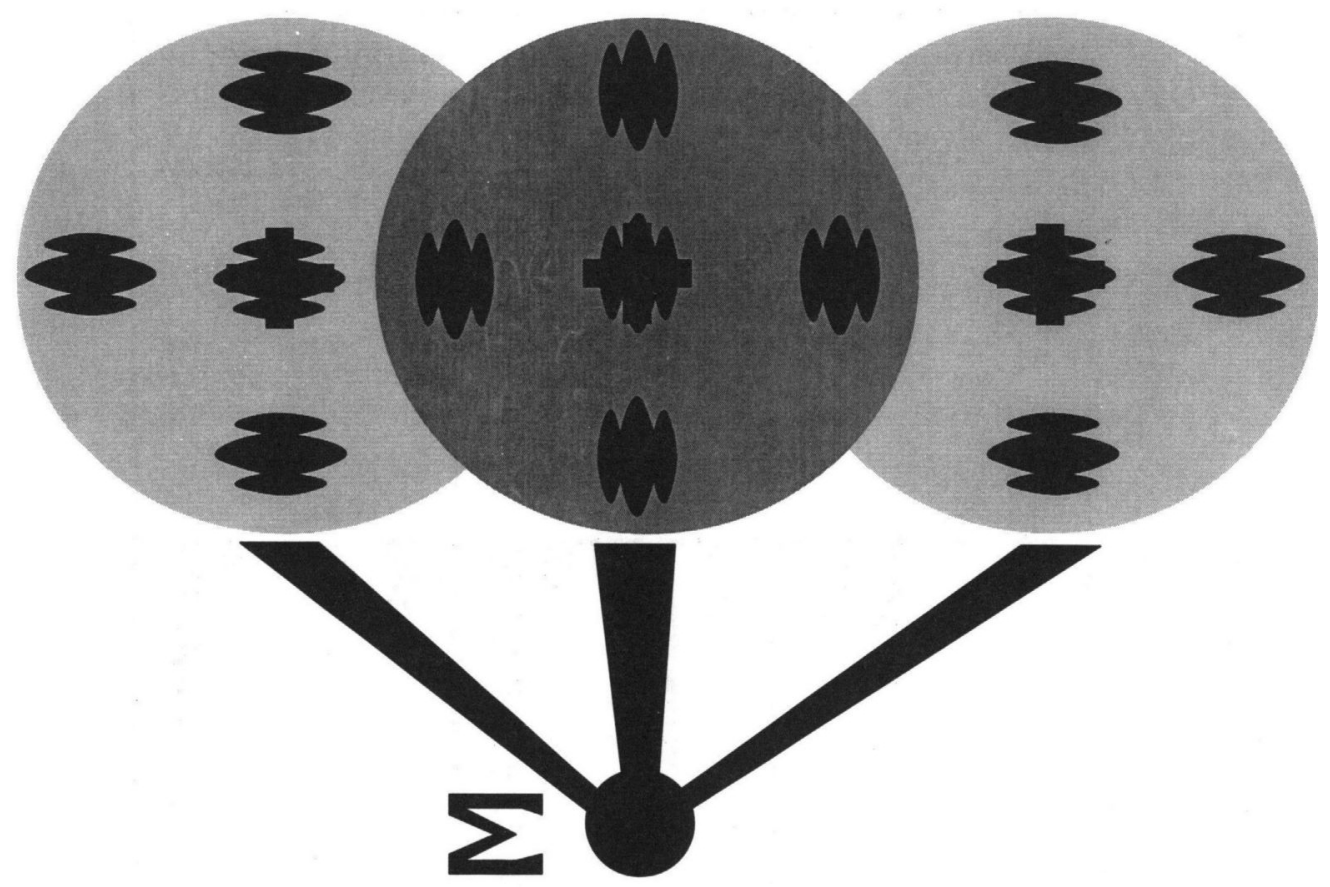

Figure 8. The proposed second-stage filter which operates on first-stage filter-response maps. This filter has Gaussian-like excitatory intramap connections, with isotropic connections that span adjacent and more distant filters (at least $7 \mathrm{deg}$ of visual arc). These resultant integrated responses can be additionally excited by similar responses but from first-stage orthogonal filters, whose spatial proximity are to the sides (1-2 deg) of the local orientation.

whether this behavior is a result of interaction between two filter-response maps (vertical and horizontal) or rather just of one. To determine this, we ran contrast gradient tasks using Gabor patches of one orientation (vertical), thus isolating only one filter-response map.

The results of contrast gradient tasks (Fig. 4) show that performance generally decreases as texture frequency increases (some curves are rather flat, but certainly the opposite behavior or positive curves are not found). This indicates two types of connections in the visual system. First, it appears that because increasing the texture frequency, but not the number of target texels, changes the psychophysical performance level, large target clusters must be processed differently than smaller ones (within a particular orientation/spatial-frequency map). Because performance does not level off in many cases for texture frequencies as low as $1 / 2$ cycles/texel (see Figs 2, 4, and 6), it is proposed that there is an integration effect and that it reaches at least to this extent (seven texel-long clusters). This differential treatment of different size clusters adds evidence to the idea that more distant interactions (not just between adjacent filters) exist, otherwise performance levels would remain the same for all texture frequencies. These interactions appear to be excitatory, with larger target clusters (smaller texture frequencies) giving rise to highest performance. The central darker circle of Fig. 8 shows a schematic diagram of how first-stage filters, selective for the same orientation and spatial frequency, might be connected, with the central filter 
(shown in the figure as vertical with a big ' + ') receiving excitatory influence from neighboring filters (also vertical, indicating intramap connections) and less from more distant ones. Smaller + signs indicate less excitatory influence. Although the envelope is shown only to be three filters wide in the figure, it extends at least to seven filters. Hence, the visibility of a foreground texel, represented by activation of the central filter of the figure, can be supplemented by additional neighboring texels of the same type in the periphery. Notice that in some cases, high-contrast targets produced flat curves, indicating that the contrast response was perhaps at a saturated level, rendering texture frequency a negligible variable in the task. This would be represented in the figure by the central filter producing such a strong response that any additional excitatory influence by like-texels (i.e. high contrast) in the periphery would contribute in a proportionately insignificant way.

Also implied by the results of contrast gradient tasks is an integration effect that is not direction-specific within any particular map, i.e. target texels aligned in parallel are no easier to see than texels orthogonally arranged. This result is different from those of orientation gradient tasks; hence, orientation gradient tasks must involve multiple filter-response maps (vertical and horizontal) and the corresponding interconnections between these maps must somehow be responsible for this asymmetric global-local interaction. This behavior is depicted in Fig. 8 by the darker circle, representing intramap (vertical) integration, receiving strong additional excitatory influence from integrated responses of orthogonal filters (horizontal) positioned at the sides of the local orientation (vertical). Hence, the proposed second-stage filter integrates a first-stage filter response (e.g., the central vertical one) with neighboring like-filter responses and/or neighboring orthogonal filter responses positioned at the sides (orthogonal texels in superposition were not considered here).

This design is representative of human behavior because for orthogonal arrangements, a high texture frequency of $1 / 2-1 / 4$ cycles/texel (with $1 / 6$ cycles/texel depicted by the cluster of three central filters in Fig. 8) is more visible than lower frequencies (multiple vertical texels; e.g., rotate Fig. $1 \mathrm{e} 90 \mathrm{deg}$ ). Notice that multiple vertical texels (orthogonally arranged) would be integrated by the dark central circle, but by introducing an orthogonal filter response at the sides, a stronger response results. For clarity, the orthogonal maps (light circles) are positioned for an optimal texture frequency of $1 / 6$ cycles/texels; however, the reader may imagine the orthogonal maps positioned closer, as the real second-stage filter would be. Also, the filter design implies that this high texture frequency with orthogonally arranged target texels would be more visible than the same foreground but in an aligned arrangement, because no excitatory orthogonal filters are positioned along the parallel virtual line running through the central filters (top to bottom). Performance curves (see Fig. 2) also demonstrate this result for most cases. The saturation performance depicted in Fig. 2 (lower graph of RC, upper graph of BR) along with the high visibility of low texture frequencies positioned in alignment (see Fig. 1c) indicates that this distant neighbor excitatory influence is relatively dominant, rendering all other texture frequencies (with either global orientation) less visible by comparison. The only exception would be the orthogonally arranged high texture frequency which from visual inspection of Fig. 2, appears to be equally visible.

A general remark concerning this schematic representation is that it can be physically rotated $90 \mathrm{deg}$, depending on the orientation of the texel (cluster) that is being 
considered (the figure presently is acting upon a vertical texel, via a first-stage filter, and thus is calculating its second-stage response by analyzing the neighboring filters). The filter is also not meant to be restricted to calculating visibility of only foreground texels, but for consistency must also be able to act on all parts of the stimulus. Because all backgrounds of stimuli used here contain the same feature, without any gradients, activity would be identical for all, being determined exclusively by intramap excitatory interactions. Also, this second-stage filter is not necessarily limited to vertically and horizontally selective filters or connections between them, with other filter-types yet to be explored. As an example, filter-response maps differing by $45 \mathrm{deg}$ might have unique interactions.

Psychophysical experiments were also performed with spatial-frequency gradient stimuli, producing performance levels that decrease as texture frequency increases (most curves are rather flat, indicating weaker interactions). Because the contrast gradient tasks give rise to filter activity within only one spatial-frequency map and their psychophysical results are similar to those using spatial-frequency gradients, we can explain this result simply with intramap connections, as exhibited by the Gaussian excitatory sensitivity envelope of the vertical filters of Fig. 8. Hence, different spatial-frequency maps do not appear to have any significant or unique interconnections. There is one interesting result that shows higher performance levels for stimuli having background texels comprised of 2 cpd Gabor patches as opposed to those with $16 \mathrm{cpd}$ patches. This result might be attributed to a visual system that has low-spatial-frequency maps which are relatively less noisy (i.e. less internal noise) as compared to higher-spatial-frequency maps. According to Rubenstein and Sagi (1990), a target's visibility is dependent on the noise content of the background. Their idea is based on reducing the discrimination task to a signalin-noise task, with less noisy backgrounds giving rise to greater target visibility. In this case, a low-frequency background ( $2 \mathrm{cpd}$ ) which is less noisy will produce higher performance levels than the reversed case (i.e. a background of $16 \mathrm{cpd}$ patches).

To summarize, the dimensions of this second-stage filter (Fig. 8) are such that there exists excitatory Gaussian-like intramap interactions that can extend up to at least seven filters (only four shown here). All of these resultant smoothed maps are in turn connected to like-maps of the orthogonal orientation, whose spatial proximity is to the sides of the local orientation and approximately one to two filters away (circles are separated by two filters here). Pilot studies of these experiments used texels of different spatial frequencies, sigma of the Gaussian envelope, and spatial proximity to adjacent texels, and it was determined that the visual angle of the foreground clusters appears to be the critical quantity which gives rise to this most visible high texture frequency. Thus, because each stimulus texel represents approximately $1 \mathrm{deg}$ of visual arc, first-stage filter dimensions within the second-stage filter of Fig. 8 would be about the same. Another interesting behavior exhibited by the data was that during the course of performing the orientation gradient task, observers' preference for high texture frequencies (i.e. when the global orientation of the foreground region is orthogonal to the local texel orientation) appeared to lessen, while lower texture frequencies became more visible. This behavior (although not seen dramatically for all observers) seems to imply that the second-stage filter has some form of plasticity. It appears as if the visual system is learning more intently to do tasks 
that are originally difficult, and after some time, this 'compensation' effect reduces orthogonal influences and allows intramap connections to dominate.

The second-stage filter described here would support a maximal response for local orientation gradients, in agreement with the experimental results of Nothdurft (1985). Sensitivity to orientation gradients does not necessarily imply a mechanism that integrates information across different orientations; however, such mechanisms were already considered theoretically (Nothdurft, 1985; Malik and Perona, 1990; Landy and Bergen, 1991). Both Malik and Perona (1990) and Landy and Bergen (1991) propose inhibitory connections between orthogonal filters spatially superimposed, although Malik and Perona claim that an aggregated response of these filters is also possible. This approach is different from the proposed second-stage filter which has excitatory influences of orthogonal filters positioned to the sides of the local orientation. Also, no evidence is found here for within-map inhibition. It is possible that the performance limiting factor in the tasks used here is a stage preceding the inhibitory stage. Note that the inhibitory stage is not necessary to account for human performance. For example, Rubenstein and Sagi (1990) used stimuli with randomly oriented texels, thus creating maps that are statistically equivalent across different orientations. Using an intramap inhibition model, Rubenstein and Sagi extracted gradients to predict human behavior. This type of randomly oriented stimuli would render the orthogonal excitatory intramap connections of the proposed filter as mere Gaussian-type intramap connections. Although Rubenstein and Sagi used a secondstage filter that includes both a Gaussian-type filter followed by a gradient detector (Laplacian), it can be argued that the Gaussian-type filter (like that of the proposed filter) is sufficient for segmenting an image. This argument assumes a visual system with an energy threshold decision stage (as opposed to a gradient threshold decision stage in the Rubenstein and Sagi model), thus singling out regions with texels having energy responses that exceed (or are less than) that of the other texels.

The experiments of Van Essen et al. (1989) showed that stimuli presented with flanking lines (surrounding the target) in parallel to the target orientation reduced neuronal response relative to the case of an isolated target. This intramap inhibitory behavior appears to be in contradiction with results reported here, although the stimulus annulus extends to approximately $3 \mathrm{deg}$ in diameter while the stimuli used here (i.e. foreground cluster sizes) extend up to $7 \mathrm{deg}$. This difference in annulus size might be significant, with very small cluster sizes (less than $3 \mathrm{deg}$ ) not explored in the present experiments.

\section{Acknowledgement}

The work reported here was conducted while at the Laboratory of Vision Research, Rutgers University, New Brunswick, NJ. We would therefore like to thank the director, Bela Julesz for his support and cooperation, as well as for his comments. We would also like to thank Uri Polat for the helpful discussions. This work was also supported by the Basic Research Foundation administered by the Israel Academy of Sciences and Humanities.

\section{REFERENCES}

Beck, J. (1982). Textural segmentation. In: Organization and Representation in Perception. J. Beck (Ed.). Erlbaum, Hillsdale, N. J.

Blakemore, C. and Campbell, F. W. (1969). On the existence of neurons in the human visual system selectively sensitive to the orientation and size of retinal images. J. Physiol. Lond. 203, 237-260. 
Caelli, T. M. (1985). Three processing characteristics of visual texture segmentation. Spatial Vision 1, 1930.

Caelli, T. M. and Julesz, B. (1978). On perceptual analyzers underlying visual texture discrimination: Part I. Biol. Cybernet. 28, 167-175.

Cannon, M. W. (1990). Inhibitory interactions in suprathreshold vision. Invest. Ophthalmol. Vis. Sci. 31, 561.

Chubb, C., Sperling, G. and Solomon, J. (1989). Texture interactions determine apparent lightness. Proc. Nat. Acad. Sci. USA 86, 9631-9635.

Fogel, I. and Sagi, D. (1989). Gabor filters as texture discriminators. Biol. Cybernet. 61, 103-113.

Graham, N. and Nachmias, J. (1971). Detection of grating pattern containing two spatial frequencies: A comparison of single-channel and multi-channel models. Vision Res. 11, 251-259.

Graham, N., Beck, J. and Sutter, A. (1992). Nonlinear processes in spatial-frequency channel models of perceived texture segregation: Effects of sign and amount of contrast. Vision Res. 32, 719-743.

Gurnsey, R. and Browse, R. (1989). Asymmetries in visual texture discrimination. Spatial Vision 4, 31-44.

Julesz, B. (1962). Visual pattern discrimination. IRE Trans. Information Theory 8, 84-92.

Julesz, B. (1980). Spatial nonlinearities in the instantaneous perception of textures with identical power spectra. Phil. Trans. R. Soc. Lond. B290, 83-94.

Julesz, B. (1981). Textons, the elements of texture perception and their interactions. Nature 290, 91-97.

Landy, M. S. and Bergen, J. R. (1991). Texture segregation and orientation gradient. Vision Res. 31, 679691.

Levitt, H. (1971). Transformed up-down methods in psychoacoustics. J. Acoustical Soc. Am. 49, 467-477.

Malik, J. and Perona, P. (1990). Preattentive texture discrimination with early visual mechanisms. J. Opt. Soc. Am. A7, 923-932.

Northdurft, H. C. (1985). Sensitivity for structure gradient in texture discrimination task. Vision Res. 25, 1957-1968.

Polat, U. and Sagi, D. (1993). Lateral interactions between spatial channels: Suppression and facilitation revealed by lateral masking experiments. Vision Res. 33, 993-999.

Rubenstein, B. S. and Sagi, D. (1990). Spatial variability as a limiting factor in texture-discrimination tasks: Implications for performance asymmetries. J. Opt. Soc. Am. A7, 1632-1643.

Sagi, D. (1990). Detection of an orientation singularity in Gabor textures: Effect of signal density and spatial-frequency. Vision Res. 30, 1377-1388.

Sagi, D. and Hochstein, S. (1985). Lateral inhibition between spatially adjacent spatial frequency channels? Percept. Psychophys. 37, 315-322.

Sagi, D. and Julesz, B. (1987). Short-range limitation on detection of feature differences. Spatial Vision 2, $39-49$.

Treisman, A. (1985). Preattentive processing in vision. Computer Vision, Graphics Image Process. 31, 156 177.

Van Essen, D. C., De Yoe, E. A., Olavarria, J., Knierim, J., Fox, J. M., Sagi, D. and Julesz, B. (1989). Neural responses to static and moving texture patterns in visual cortex of the macaque monkey. In: Neural Mechanisms of Visual Perception. D. M. K. Lam and C. Gilbert (Eds). Portfolio Publications, The Woodlands, TX, pp. 137-154. 